This is a peer-reviewed, accepted author manuscript of the following article: Al Ameri, J., Alsuraifi, A., Curtis, A., \& Hoskins, C. (2020). Effect of poly(allylamine) molecular weight on drug loading and release abilities of nano-aggregates for potential in cancer nanomedicine. Journal of Pharmaceutical Sciences. https://doi.org/10.1016/j.xphs.2020.06.018

\title{
Effect of poly(allylamine) molecular weight on drug loading and release abilities of nano-aggregates for potential in cancer nanomedicine
}

\author{
Jenan Al Ameri ${ }^{1,2}$, Ali Alsuraifi ${ }^{1,3}$, Anthony Curtis ${ }^{1}$, Clare Hoskins ${ }^{1,4 *}$
}

1. School of Pharmacy and Bioengineering, Keele University, Keele, Staffordshire, UK, ST5 5BG

2. Department of Chemistry, College of Science, University of Basrah, Basrah, Iraq

3. College of Dentistry, University of Basrah, Basrah 61004, Iraq

4. Department of Pure and Applied Chemistry, University of Strathclyde, Glasgow, UK, G1 1RD

*Corresponding author: Dr Clare Hoskins, clare.hoskins@strath.ac.uk, +44141 5482796

\begin{abstract}
Poly(allylamine) graft polymers have been shown to hold potential as drug delivery vehicles and complexation agents for biological molecules such as insulin. The nanoparticles formed upon aggregation or complexation allow for enhanced cellular trafficking resulting in enhanced efficacy. Multiple reports have shown the ease of synthesis and reliability of these graft polymers, however, little investigation into the effect of the molecular weight of the homopolymer poly(allylamine) has been carried out. In this work we synthesized a range of oxadiazole grafted poly(allylamine) derivatives of varied molecular weight $(15,17.5,120$ \& $900 \mathrm{kDa}$ ) set at a 5\% polymer:oxadiazole mole grafting. The effect of molecular weight on the size, critical aggregation concentration and drug loading / release was evaluated in model drugs before loading the optimal formulation with doxorubicin and carrying out a preliminary cytotoxicity study. In line with other cationic polymers, the larger poly(allylamine) amphiphilic derivatives resulted in greater drug loading, however, the particle size increased whilst drug loading dramatically decreased, which for cancer nanomedicine could be a barrier for pharmaceutical use.
\end{abstract}

Keywords: Amphiphilic polymer, molecular weight, hydrophobic drug, drug delivery, graft polymer, drug release 


\section{Introduction}

Amphiphilic polymers have played a key role in the development of advanced drug delivery technologies by controlling solubility, release, permeability and stability of active ingredients. Polymeric nano-aggregates can be created from a diverse range of architectures and form a plethora of structures in the aqueous environment including vesicles, polymeric micelles, and nanoparticles [1]. Graft polymers are composed of a water soluble homopolymer backbone with hydrophobic groups randomly grafted on $[2,3]$. The resulting amphiphilic comb-shaped molecules aggregate through weak non-covalent hydrophobic-hydrophobic interactions in aqueous environments, where the hydrophilic moiety will remain in association with the aqueous phase whilst the hydrophobic moieties will 'shield' themselves, thereby forming a micellar core-shell system.

Amphiphilic polymers possess the ability to encapsulate hydrophobic drug molecules inside their hydrophobic core, hence increasing the aqueous solubility of the drug. In vivo a hydrophilic protein corona forms surrounding the aqueous shell of the self-assembly. This is believed to be a contributing factor to the increased stability experienced by these nanoaggregates in blood plasma resulting in increased circulation times and reduced opsonisation and capture by the reticulo-endothelial system. Polymeric aggregates are well-known to possess a lower critical micelle concentration (CAC) compared with surfactant micelles. These properties make polymeric nano-aggregates excellent candidates for drug solubilisation and delivery, particularly for bulky anticancer drugs [4]. However, studies have shown, that in order for nanoparticles to effectively permeate cancer microenvironments, the particle size must be preferentially within the nano-size range [5].

Poly(allylamine) (PAA) is a positively charged long chain polymer which has previously been investigated for its use as an aqueous homopolymer backbone for graft polymer fabrication $[6,7]$. PAA is available clinically in its cross-linked state as an oral phosphate binder. PAA can undergo chemical modification at its primary amine functional group sites enabling grafting of a multitude of hydrophobic moieties via simple nucleophilic substitution reactions. Thompson and colleagues have reported that varying the hydrophobic groups on the PAA backbone had an important impact on the polymer properties and the self-assemblies formed in the aqueous environment [8].

However, the effect on aggregation ability and solubilisation potential of varying the molecular weight PAA homopolymer backbones have not been studied. Hence, in this work, we will develop a series of amphiphilic PAA polymers modified with a hydrophobic oxadiazole pendant group. We will vary the molecular weight of the homopolymer backbone whilst maintaining 5\% molar pendant substitution. Oxadiazole (5-(4-chlorophenyl)-1,3,4-oxadiazole2-thiol) has been identified as a promising hydrophobic pendant group due to the thiol functionality which can be subsequently exploited to bind gold colloids conferring imaging capabilities. In this study two model hydrophobic drugs (propofol and griseofulvin) will be investigated in order to estimate whether the amphiphiles created can be used for drug solubilisation, followed by incorporation of anticancer agent doxorubicin (DOX). Propofol is an intravenous sedative discovered in 1977 and approved for use in humans by the FDA in 1989. Propofol has not yet been earmarked as a controlled drug anywhere in the world except in Korea [9] even though there is the possibility to obtain and abuse it as witnessed in a number of high-profile cases worldwide. It is a small hydrophobic molecule that is currently 
administered as an oil-based formulation. This is problematic because it causes pain on injection, hence an aqueous based formulation would be much more desirable. Griseofulvin was discovered in 1958. It is insoluble in water but sparingly soluble in acids such as acetic acid, and in low molecular weight alcohols such as methanol and ethanol. Griseofulvin is used in the treatment of many dermatological diseases such as delicate skin and nails as well as scalp and the systematic treatment of inflamed lesions. The solubility of griseofulvin in water is very low, explaining the incomplete absorption of the drug after oral administration. Several factors affect the absorption of the drug within the body such as fat intake, structure, particle size and the rate of dissolution [10]. The solubility of griseofulvin is extremely important for its efficacy. Therefore, better formulations are required to ensure dose regularity both intra and inter patients. DOX is an anticancer agent whose poor physicochemical properties and extremely high toxicity require careful formulation before administration [11]. It has been shown to be effective in a number of cancers such as liver [12], breast [13] and pancreatic [14]. In this study we will incorporate the DOX into the optimal formulation parameters deduced using the model drugs, and subsequently study the efficacy enhancement after exposure to pancreatic cancer cell lines.

The aim of this study was to determine the influence of homopolymer molecular weight on the ability of the nano-aggregates to act as efficient drug solubilizing agent for a nanoformulation of DOX. The was investigated via fabrication of a range of PAA based amphiphilic combshaped polymers. The polymers were synthesized and chemically characterized using nuclear magnetic resonance (NMR) and Fourier transform infrared (FTIR) spectroscopy. Ability of the polymers to aggregate in aqueous environments was determined using photon correlation spectroscopy and surface tension measurement. Ability of the amphiphiles to encapsulate hydrophobic drugs was determined using model drugs propofol and griseofulvin and measured using high performance liquid chromatography (HPLC). The influence of molecular weight of the homopolymer backbone on drug solubilization and release was determined, followed by a preliminary cytotoxicity study in pancreatic cancer cell lines.

\section{Experimental}

Griseofulvin was purchased from Alfa Aesar, UK and propofol from Sigma Aldrich, UK. DOX was purchased from LC Laboratories (Woburn, USA). Deuterated methanol was purchased from Cambridge Isotope Laboratories Inc., USA. All other chemicals were purchased from Sigma Aldrich, UK and reagents from Fisher Scientific, UK. Dialysis tubing was purchased from Medicell International Ltd, UK. Syringe filters were purchased from Whatman, UK.

\section{PAA-Ox5 Synthesis}

PAA of varied molecular weight $(15,17.5,120 \& 900 \mathrm{kDa})$ was modified in 1:0.05 molar feed ratio with 5-(4-chlorophenyl)-1,3,4-oxadiazole-2-thiol (Oxadiazole, Ox) (monomer: pendant) (Figure 1). The hydrochloride salt of poly(allylamine) was first converted into the free base form via addition of a methanol:water solution of sodium hydroxide. This was then purified using dialysis in a $7 \mathrm{kDa}$ visking membrane against water for $24 \mathrm{~h}$. After this time the polymer was freeze dried. The solid PAA free base $(2 \mathrm{~g})$ was dissolved in a $50 \mathrm{~mL}$ solution of $1: 1(\mathrm{v} / \mathrm{v})$ methanol:chloroform with stirring for $10 \mathrm{~min}$, subsequently $2 \mathrm{~mL}$ of trimethylamine was added. The reaction was stirred at room temperature for $0.5 \mathrm{~h}$. The oxadizaole was dissolved in $20 \mathrm{~mL}$ of a $1: 1(\mathrm{v} / \mathrm{v})$ methanol:chloroform solution and added drop wise to the polymer solution over $0.5 \mathrm{~h}$. This was stirred at $37{ }^{\circ} \mathrm{C}$ for $18 \mathrm{~h}$. The solvent was then removed on a rotary evaporator, and the polymer residue remaining was washed with $10 \mathrm{~mL}$ diethyl ether and the solvent evaporated a further 3 times. The residue was dissolved in deionised water and 
exhaustively dialysed in visking membrane 12-14 $\mathrm{kDa}$ against deionised water for $24 \mathrm{~h}$. The polymer was then freeze dried and stored in an airtight container.

\section{Chemical characterisation of PAA amphiphiles.}

Nuclear magnetic resonance spectroscopy (NMR)

${ }^{1} \mathrm{H}$ NMR analysis of samples was carried out on all modified polymers in deuterated methanol at $25{ }^{\circ} \mathrm{C}$, utilizing a Bruker $400 \mathrm{MHz}$ Ultra shield NMR spectrometer (Bruker BioSpin, Germany). Sonication of polymers in the deuterated methanol was carried out on a Soniprep 150 (MSE Ltd, UK) in order to ensure they were completely dissolved in the solvent. Spectra were compared to the PAA before modification, to confirm synthesis was successful.

\section{Fourier transform infrared spectroscopy}

Fourier Transform Infrared Spectroscopy (FTIR) was carried out for all of the modified polymers using a Perkin Elmer iS10 instrument (Spectrum BX, UK), fitted with a diamond powder tip attenuated total reflectance attachment. The polymers were placed under the diamond tip and 20 scans were run followed by background correction. Spectra were compared to the PAA before modification, to confirm synthesis was successful.

\section{Physical characterisation of PAA amphiphiles \\ Critical aggregation concentration $(C A C)$ determination using surface tension measurement}

Polymers of varied concentrations $\left(0.0195 \mathrm{mgmL}^{-1}-5 \mathrm{mgmL}^{-1}\right)$ were prepared using water as the diluent and sonicated for $10 \mathrm{~min}$ before cooling to room temperature. The surface tension of polymer solutions was measured at $25^{\circ} \mathrm{C}$ using a torsion balance (OS, White Electrical Instrument $\mathrm{Co}$, London). The platinum ring was cleaned with ethanol and distilled water prior to analysis of each sample. The measurement was recorded in triplicate for each polymer solution to get an average value. The surface tension was plotted against polymer concentration to allow for the estimation of CAC which was indicated via a deviation from the CAC of water.

\section{Photon correlation spectroscopy and zeta potential measurement}

Hydrodynamic diameters and zeta potential were determined using photon correlation spectroscopy on a Zetasizer Nano-ZS (Malvern Instruments, UK). Polymers were diluted in deionised water to make $1 \mathrm{mgmL}^{-1}$ solutions and sonicated for $30 \mathrm{~s}$ before analysis at $25{ }^{\circ} \mathrm{C}$.

\section{Drug loading}

Polymer amphiphiles were dissolved in deionised water $\left(1 \mathrm{mgmL}^{-1}, 3 \mathrm{mgmL}^{-1}\right.$ and $\left.6 \mathrm{mgmL}^{-1}\right)$ with probe sonication for $10 \mathrm{~min}$. Drug (propofol or griseofulvin) was added at 1:1, 5:1 and 10:1 initial drug:polymer feed ratios and the drug-polymer solutions were probe sonicated for a further $10 \mathrm{~min}$. After cooling to room temperature, the solutions were filtered using $0.45 \mu \mathrm{m}$ syringe filters to remove any unencapsulated drugs.

\section{Drug Quantification}

\section{Quantification of Propofol}

The drug loading capacity of the self-assemblies was determined using high performance liquid chromatography (HPLC) (Shimadzu prominence UFLC, UK). A RP Zorbax ODS $250 \mathrm{~mm} x$ $46 \mathrm{~mm}$ x $5 \mu \mathrm{m}$ HPLC column (Hichrom, UK) was used with flow rate of $1 \mathrm{~mL} \mathrm{~min}^{-1}$ (80:20 $\mathrm{v} / \mathrm{v}$ methanol :water) in an isocratic mode. The samples were diluted with mobile phase and 20 $\mu \mathrm{L}$ was injected onto the column. The amount of propofol present in the samples was determined by comparing to a standard calibration carried out previously with propofol dissolved in methanol $\left(4 \mu \mathrm{gmL}^{-1}-250 \mu \mathrm{gmL}^{-1}\right), \mathrm{R}^{2}=0.9988$. 


\section{Quantification of griseofulvin}

The drug concentration was measured on a RP Phenomenex C18 $250 \mathrm{~mm}$ x $46 \mathrm{~mm}$ x $5 \mu \mathrm{m}$ HPLC column and detected at $293 \mathrm{~nm}$ (Shimadzu prominence UFLC, UK). The mobile phase $(45: 55 \mathrm{v} / \mathrm{v})$ acetonitrile: $45 \mathrm{mM}$ potassium dihydrogen phosphate made up in water and $\mathrm{pH}$ adjusted to $\mathrm{pH} 3$ with orthophosphoric acid) ran at a rate of $1 \mathrm{~mL} \mathrm{~min}^{-1}$. The samples were diluted with mobile phase and $20 \mu \mathrm{L}$ was injected onto the column, the resultant peak with a retrntion time of 9.5 min was analysed. The quantification of griseofulvin present in the samples was determined from a standard calibration of griseofulvin in mobile phase $\left(0.6 \mu \mathrm{gmL}^{-}\right.$ $\left.{ }^{1}-10 \mu \mathrm{gmL}^{-1}\right), \mathrm{R}^{2}=0.9987$.

\section{In vitro drug release from nano-aggregates}

The drug loaded polymers $(2 \mathrm{~mL})$ were pipetted into a dialysis tube (MW cut off $=12-14 \mathrm{kDa})$ and dialyzed against $200 \mathrm{~mL}$ deionised water (to mimic sink conditions) for $24 \mathrm{~h}$ with stirring at $37{ }^{\circ} \mathrm{C}$. At various timepoints over the $24 \mathrm{~h}$ study duration $1 \mathrm{~mL}$ of the external deionised water was periodically removed and replaced with $1 \mathrm{~mL}$ of fresh deionised water. The amount of the released drug was determined using HPLC via analysis of the aliquoted samples as a percentage of total drug encapsulated.

\section{DOX formulation and in vitro evaluation Loading and quantification of $\mathrm{DOX}$}

DOX was loaded into the polymer aggregates at $6 \mathrm{mgmL}^{-1}$ polymer concentration and 10:1 drug:polymer ratio. The drug concentration was quantified by reverse phase high performance liquid chromatography RP-HPLC (Prominence, DEGASSER, LC20AD, SHIMADZU) utilizing a fluorescence indicator at $480 \mathrm{~nm}$ (Excitation) and $560 \mathrm{~nm}$ (Emission) (Jasco, PU980, Japan; column C18(2), $150 \mathrm{~mm} \times 4.60 \mathrm{~mm} 5$ micron, flow rate: $1 \mathrm{mLmin}^{-1}$, injection volume: $20 \mu \mathrm{L}$ ). The buffer applied in HPLC mobile phase was arranged by adding $10 \mathrm{mmoL}$ ammonium hydrogen phosphate (to boost the retention) and $5 \mathrm{~mL}$ trimethyl amine to $200 \mathrm{~mL}$ of deionised water. Then the $\mathrm{pH}$ of the solution was adjusted to 4.0 with orthophosphoric acid. The mobile phase was a mixture of buffer $\mathrm{pH}$ 4.0/acetonitrile/methanol (60:24:16, V/V/V), sonicated for $10 \mathrm{~min}$ (Toshniwal ultrasonic cleaner, India) and filtered through $0.22 \mu \mathrm{m}$ filter. $\mathrm{R}^{2}=0.9988$. the regression equation obtained is: $\mathrm{y}=7.524 \mathrm{X}+0.0573$.

\section{MTT([3-(4,5-Dimethylthiazol-2-yl)2,5DiphenyltetrazoliumBromide]) cytotoxicity assay}

Human pancreatic adenocarcinoma BxPC-3, cells (150000 cells/well) were seeded into a 96well plate and incubated at $37^{\circ} \mathrm{C}$ with $5 \% \mathrm{CO} 2$ for $24 \mathrm{~h}$. A $20 \mathrm{mgmL}^{-1}$ stock solution was prepared of the DOX formulation (and free drug). From this stock solution eight dilutions $(0.1$ $\mathrm{mgmL}^{-1}-1 \times 10-5 \mathrm{mgmL}^{-1}$ ) using media as the diluent were prepared After $24 \mathrm{~h}$, the formulations were removed and replaced with fresh media $(100 \mu \mathrm{L})$. MTT 3-[4, 5dimethylthiazole-2-yl]-2, 5-diphenyl tetrazolium ( $50 \mu \mathrm{L}, 5 \mathrm{mgmL}-1$ in PBS) was added to the wells and then the plate was incubated $\left(37^{\circ} \mathrm{C}\right.$ with $\left.5 \% \mathrm{CO} 2\right)$ for $4 \mathrm{~h}$. The MTT was then removed from the wells. The purple formazan was remaining complexes were dissolved in $(100 \mu \mathrm{L}) \mathrm{DMSO}$, and absorbance recorded at $570 \mathrm{~nm}$ using a microplate reader (Tecan, infinite 200 pro, GmbH 5082, Australia). Percentage cell viability was calculated relative to the negative and positive controls (no cell treatment and complete cell lysis using $2 \%$ Triton-X). All studies were run in triplicate $(n=3)$ and the average values were recorded. 


\section{Results and Discussion}

\section{Synthesis and Characterisation of PAA-Ox5}

PAA-Ox 5 polymers were fabricated through a facile substitution reaction of the primary amines in the parent PAA structure and the chloride functionality of the oxadiazole compound (Figure 1), with yields shown in Table 1. The modified polymer structure was confirmed using NMR. The NMR spectra for the different molecular weight amphiphiles compared to the parent PAA are shown in Figure 2A and SI-Figure 1. The spectra obtained from all molecular weights were comparable after oxadiazole attachment, as expected. Peaks were observed at $0.75 \mathrm{ppm}$, $2.50 \mathrm{ppm}, 3.00 \mathrm{ppm}, 1.40 \mathrm{ppm}$ and $1.50 \mathrm{ppm}$ which can be attributed to the respective $\mathrm{CH}_{2}$ and $\mathrm{CH}$ groups in the homo polymer backbone. Also, peaks observed between at 7.25-7.75 ppm are attributed to the aromatic $\mathrm{CH}$ protons located in the oxadiazole pendants. Unfortunately, it was not possibly to observe the peak arising from the proton in the - $\mathrm{SH}$ group of the oxadiazole pendant, possibly due to its relative abundance in this macromolecular system. However, the presence of the aromatic peaks which were not observed in the unsubstitued PAA alone, would suggest that pendant conjugation was successful.

In order to confirm this, FTIR analysis was carried out between $4000 \mathrm{~cm}^{-1}-650 \mathrm{~cm}^{-1}$ to detect the specific functional groups existing in the modified amphiphiles. Characteristic peaks were observed at $3279 \mathrm{~cm}^{-1}$ and $2849 \mathrm{~cm}^{-1}$ arising from the $\mathrm{N}-\mathrm{H}$ stretching and $\mathrm{C}-\mathrm{H}$ stretching in the PAA backbone as observed in Figure 2B and SI-Figure 2. Peaks observed at $1580 \mathrm{~cm}^{-1}$ indicated that hydrophobic pendant attachment had been successful. These peaks were due to the bending and stretching vibrations of the aromatic ring $\mathrm{C}=\mathrm{C}$ located in the pendant oxadiazole moiety. These findings supported the observations and conclusions drawn from the NMR data and indicated that the modification across all the molecular weights of PAA had proceeded successfully. In 2013 Barnett et al. first reported the synthesis of a PAA bearing an oxadiaxole pendant group [3]. The structural analysis in our study agreed with their findings.

\section{Critical aggregation concentration determination via surface tension measurement}

Hydrophobic-hydrophobic interactions between the aqueous media and amphiphilic polymer are the driving forces behind aggregate formation [15]. When these amphiphiles are in aqueous solution below their critical aggregation concentration they reside at the bulk liquid-air interface. However, when the concentration is increased, the net hydrophobicity seeks to shield itself from the aqueous solvent and spontaneous core-shell aggregate formation occurs. This can be estimated using surface tension measurement, when plotted against concentration, the point of inflection indicates the point at which aggregate formation occurs. When the surface of the solution-air interface becomes saturated with amphiphilic moieties, the core-shell aggregation is energetically favoured resulting in a shift in the surface tension - hence the critical aggregation concentration is observed [16]. 
In this study the PAA amphiphiles were first dissolved in deionised water, which has a surface tension of $0.072 \mathrm{~N} / \mathrm{m}$. Figure 3 shows the surface tension measurements over the varied concentrations for the PAA amphiphiles. Here it is observed that increasing molecular weight of the polymer (at fixed molar ratio modification with oxadiazole) resulted in a decrease in $\mathrm{CAC}$ value. This is probably because at higher molecular weights the polymer has much greater flexibility and there is an increased opportunity for polymer coiling or wrapping around itself to form intramolecular aggregates. This view is strengthened when looking at the shape of the graph for both the MW $120 \mathrm{kDa}$ and $900 \mathrm{kDa}$ amphiphiles, where more than one point of inflection is observed in the graph, leading to the assumption that more than one type of aggregation is occurring - both intramolecular and intermolecular. At the lower molecular weights only one major point of inflection is observed. These findings indicate that at higher molecular weights and fixed 5\% molar grafting, the aggregates formed are likely to be more stable, with the CAC value increasing from $0.02 \mathrm{Nm}^{-1}$ to $0.04 \mathrm{Nm}^{-1}$ to $0.3 \mathrm{Nm}^{-1}$ to $0.6 \mathrm{Nm}^{-1}$ as the PAA molecular weight decreases from $900 \mathrm{kDa}$ to $120 \mathrm{kDa}$ to $17.5 \mathrm{kDa}$ to $15 \mathrm{kDa}$ respectively. Interestingly, a substantial difference was observed between $15 \mathrm{kDa}$ and 17.5 $\mathrm{kDa}$ despite these two molecular weights being relatively close. The reason for this is largely unknown, however it could be attributed to the random grafting nature of these polymers.

\section{Drug loading}

The drug encapsulation studies carried out using propofol as a model drug (Figure 4) showed that at higher polymer concentration and higher initial drug:polymer feed ratios, increased drug solubilisation was achieved. This finding is in agreement with other studies in the literature where propofol has been encapsulated inside PAA amphiphiles. The study also showed that with increased PAA molecular weight of the PAA-Ox5 increasing drug incorporation was observed in general. For example at $6 \mathrm{mgmL}^{-1}$ polymer concentration and 10:1 drug:polymer feed ratio the amount of propofol solubilised increased from $12.2 \mathrm{mgmL}^{-1}$ to $18.7 \mathrm{mgmL}^{-1}$ to $25.7 \mathrm{mgmL}^{-1}$ in the polymers with increasing molecular weight from $15 \mathrm{kDa}$ to $17.5 \mathrm{kDa}$ to $120 \mathrm{kDa}$ respectively, all of which where significantly higher than the intrinsic aqueous drug solubility $(\mathrm{p}<0.05)$. This phenomenon could be due to the aggregates forming at lower concentrations, therefore capable of greater solubilisation; or more likely, due to their lengthier chains being capable of wrapping and expansion without disruption to the aggregate structure upon drug incorporation [17]. However, at the $900 \mathrm{kDa}$ molecular weight, no drug could be detected in the PAA-Ox5 amphiphiles after filtration. This is likely to be due to the longer polymer chains forming much larger aggregate structures which simply were not capable of passing through the 0.45 micron filters - and not due to its complete inability to solubilise propofol. For cancer nanomedicine, smaller nano-aggregates are preferrential in order to be small enough to effectively manouver through the tumour microenvironment and reach their area of need [ref]. Therefore in this studt, those polymers who formed aggregates after loading which exceed the 0.45 micron range would not be considered useful for our application, and therefore the decision was taken to focus the study on the $15 \mathrm{kDa}, 17.5 \mathrm{kDa}$ and $120 \mathrm{kDa}$ polymers only.

A similar trend was observed with the loading of griseofulvin into the PAA amphiphiles (Figure 5), whereby, increasing initial drug:polymer loading ratio or increasing excipient (polymer) concentration resulted in greater drug encapsulation after filtration and a significant increase in drug solubility compared with the intrinsic griseofulvin solubility $(p<0.05)$. Again, in agreement with the propofol loading studies, increasing the molecular weight of the PAA backbone in the PAA-Ox5 resulted in notable increases in drug loading ability. As with the findings previously reported, it appeared that no drug had been encapsulated within the 900 $\mathrm{kDa}$ polymer aggregates as measured using HPLC. However, again this is likely to be due to 
the heavily loaded and core expanded aggregates being too large to pass through the 0.45 micron syringe filter, resulting in complete extraction of the aggregates and hence no detection of drug. The results from this study are in agreement with other studies carried out on the molecular weight of polymers and their influence on drug loading [18-20].

\section{Photon correlation spectroscopy and zeta potential measurement}

In order to study this hypothesis further, photon correlation spectroscopy was used to investigate the size of the aggregated species both before and after drug loading. The data (Table 2) showed that all the unloaded nano-aggregates were of similar size ranging from 117 $-160 \mathrm{~nm}$. The general trend observed was that size became slightly larger with increasing PAA molecular weight in the PAA-Ox5 amphiphiles, with exception of PAA $17.5 \mathrm{kDa}$. After loading with propofol the PAA $15 \mathrm{kDa}$ and $17.5 \mathrm{kDa}$ expanded to $173 \mathrm{~nm}$, possessing identical sizes. This trend was also observed in the griseofulvin loaded aggregates where the size of the formulations was $121 \mathrm{~nm}$ and $123 \mathrm{~nm}$, respectively. Increasing molecular weight to $120 \mathrm{kDa}$ resulted in larger aggregates, in agreement with the loading data. As more drug is incorporated, the hydrodynamic diameter of the aggregates expands in order to accommodate the larger amount of drug molecules, in this case to $187 \mathrm{~nm}$ and $196 \mathrm{~nm}$ for propofol and griseofulvin respectively. Interestingly, when the molecular weight of the PAA component in the PAA-Ox5 was increased to $900 \mathrm{kDa}$, no size data could be obtained using the nanosizer with sample errors being reported. This occurred in both samples. The reason for this, in accordance with the loading data, may be explained by the lack of aggregates remaining in solution. As the 900 $\mathrm{kDa}$ amphiphiles loaded the drug in large quantities, their aggregates would be expected to drastically increase in size, and if these were unable to pass through the filter would result in being removed from the solution, hence leaving only water as the diluent behind.

Further analysis of these samples was carried out using zeta potential measurement (Table 2). Here, surface charge of the loaded and unloaded vehicles was carried out. Theoretically, by increasing PAA molecular weight in the backbone of the PAA-Ox5, the surface charge should increase, due to the increase in primary amines in the cationic polymer. However, in reality large shifts were not observed, this can be attributed to the wrapping of the polymers forming aggregates of similar sizes in their unloaded form across the range of molecular weights, therefore exposing similar amounts of primary amines to the exterior of the core-shell structure. This was reflected in the similar zeta potential measurements ranging from $+16 \mathrm{mV}$ to +22 $\mathrm{mV}$. PAA amphiphiles have previously been reported to form micellar aggregates in aqueous environments, on this basis, after drug loading no major shift in surface charge was expected, this is due again to the exposed surface of the aggregate remaining similar to the unloaded aggregates. Our data confirmed that the surface charge both before and after loading in the PAA-Ox5 aggregates with those amphiphiles consisting of a PAA backbone of $15 \mathrm{kDa}, 17.5$ $\mathrm{kDa}$ and $120 \mathrm{kDa}$ are uniformly similar. However, this trend was not observed for the $900 \mathrm{kDa}$ amphiphile: with both the propofol and grisoefulvin samples, the surface charge data dramatically reduced down to $0 \mathrm{mV}$ and $+1 \mathrm{mV}$ respectively. Looking at this data in conjunction with the other studies, we can estimate that the reason for such a shift is due to the aqueous solvent no longer containing aggregates which have been removed on filtration. Interestingly, in this instance, the zetasizer did not flag a problem with the sample, as it had with the size measurement, but this is expected to be due to the subtle differences in measurement technique between the parameters. This data confirmed the theory that the highest molecular weight $900 \mathrm{kDa}$ PAA amphiphiles were no longer present in solution. Therefore, it was decided to carry out no further work with these samples moving forward. 


\section{In vitro drug release}

Investigation into drug release parameters is of high importance for any new excipient system. Previously scientists have reported a linear correlation between the drug release and the absorption in the body [21]. In order to elucidate the effect of molecular weight of the homopolymer backbone on amphiphile aggregate drug release, the formulations were dialysed against water to mimic the vast dilution gradient and sink conditions experienced after administration in the body [22].

The drug release study of propofol from the PAA-Ox5 amphiphiles shown in Figure 6A indicates that the higher the molecular weight of the polymer backbone, the slower the drug release rate: Only $35 \%$ of propofol was released form formulation after $72 \mathrm{~h}$ and $50 \%$ for PAA $17.5 \mathrm{kDa}$ which where both significantly less than the $73 \%$ released from PAA $15 \mathrm{kDa}$ aggregates over the same time period $(\mathrm{p}<0.05)$. The ability of drug to release from a carrier system is also as important as its ability to become encapsulated, particularly for cancer nannomedicine. There is no use solubilising anticancer drugs for delivery, if it is energetically more favourable for them to reside within the lipophilic core of the aggregate, never to be released to exert their pharmaceutical effect. On the other hand, often long acting or sustained release of compounds which are eliminated or metabolised too rapidly may be required and in these instances a longer release profile would be advantageous. Although this study was used to evaluate the effect of PAA molecular weight on drug incorporation and release, the application of the technology moving forward is for cancer nanomedicine. As discussed previously the two drugs being used were model drugs to give an indication of aggregate performance, ultimately for DOX encapsulation. Hence, after examining the drug release study, it was decided only to determine the release profile from griseofulvin using only the optimum polymer from the propofol study, as an iterative step in assessing the one optimal lead polymer system which would be taken forward for DOX solubilisation. This was deemed to be PAA-Ox5 containing the $15 \mathrm{kDa}$ PAA backbone. Figure 6B shows the release of griseofulvin from the PAA-Ox5 aggregates. Here, it can be observed, that although the $15 \mathrm{kDa}$ system released the propofol the most rapidly, very little griseofulvin was released over a similar time period (less than $0.5 \%$ over $72 \mathrm{~h}$ ). This highlights the importance of trialling these systems with more than one drug molecule, as although they may universally solubilise, albeit to different extents, the aggregates can often release via different release profiles. The drug loading studies showed that much less griseofulvin had been incorporated into the hydrophobic core compared with propofol. However, griseofulvin is a much larger and more hydrophobic drug molecule, therefore steric hindrance is likely to be the limiting factor for incorporation [23]. However, its greater hydrophobicity is the reason that minimal drug release is observed. This is because it is energetically more favourable for the highly hydrophobic molecule to reside within the lipophilic core of the polymeric aggregates (despite them existing in dynamic equilibrium) than expose themselves to the aqueous phase [24]. Therefore, in the case of griseofulvin, if a more rapid release profile was desired, it is suggested further decreasing the PAA molecular weight in the PAA-Ox5.

\section{DOX formulation and in vitro evaluation}

Based in the data obtained with the model drugs, DOX was loaded into the PAA-Ox5 $15 \mathrm{kDa}$ at $6 \mathrm{mgmL}^{-1}$ polymer concentration and 10:1 drug:polymer ratio. HPLC analysis determined that up to $3.3 \mathrm{mgmL}^{-1}$ DOX was loaded into the aggregates, with up to $30 \%$ release observed after $24 \mathrm{~h}$ (Figure 7A). Given that DOX is a bulky molecule, the level of loading and indeed slow release were surprising using PAA-Ox5. These findings strengthen the rationale, that the shortest polymer molecular weight was the most suitable for the cancer nanomedicine system, as further reducing the release rate could be detrimental in cancer therapy, where drug 
resistance can occur when cells are exposed to low concentrations of drug over sustained time periods. Our preliminary in vitro study showed that after $24 \mathrm{~h}$ incubation with pancreatic cancer cell lines, the polymer itself did exhibit some toxic nature. This is not wholey unsurprising due to its highly cationic nature, and in line with previous reports [1,3,5]. After $24 \mathrm{~h}$ the DOX did not appear to possess an $\mathrm{IC}_{50}$ value over the concentrations tested, whilst the formulation possessed an $\mathrm{IC}_{50}$ of around $0.05 \mathrm{mgmL}-1$. This increase in toxicity can be attributed either to an additive effect from the polymer excipient, or due to the more rapid DOX internalisation into cells after formulation, the exact reason has yet to be explored. Nevertheless, the study does confirm that formulating DOX into the PAA-Ox 5 amphiphiles does not hinder its activity.

\section{Conclusion}

The aim of this study was to examine the effect of molecular weight of PAA on its oxadiazole conjugated amphiphilic derivatives ability to solubilise and release drug molecules from the aggregates formed in aqueous environments, with aim to be used for further studies in cancer nanomedicine. Although other studies have examined such parameters in other cationic polymers, this has not been studied in PAA. PAA has proven itself to be capable of acting as a universal drug solubilising agent which holds potential for the delivery of stubborn hydrophobic compounds. The driving force for aggregate formation is via a reduction in Gibbs' free energy. We hypothesised that increasing the cationic backbone in the PAA-Ox5 amphiphiles would result in greater drug solubilisation, as the longer chains of the polymers would accommodate greater drug quantities without disrupting the hydrophobic-hydrophobic forces keeping the core-shell aggregate intact. Our findings showed that this hypothesis was correct, which agrees with other studies of different homopolymers in the literature used for graft amphiphilic polymers. However, at the highest molecular weight tested of $900 \mathrm{kDa}$, it is proposed that the aggregates formed have enlarged to such an extent, heavily laden with drug, that they enter a size range outwith the use as a cancer nanomedicine and therefore, were discarded from the study. They could be used however in other systems where size is not of paramount importance such as oral drug delivery.

The drug release from nanoparticles depends on many factors such as diffusion from the polymer nanoparticles, desorption of the surface drug polymer nanoparticle and a combined degradation/diffusion process. Drug release and biodegradation are important in the development of drug delivery systems. In this work, the drug release was influenced by polymer molecular weight and drug loading. Polymers with higher molecular weight tend to possess slower drug release profiles compared to those with lower molecular weight. Taking our findings into account, looking at the surface tension data, the aggregates formed at high molecular weight are likely to be more stable upon dilution, and incorporate greater drug quantities; however, drug release would be very slow and may not be fit for purpose in chemotherapy, where this may induce drug resistance. Therefore, based on this, we used PAA as $15 \mathrm{kDa}$ for the solubilisation of DOX in order to evaluate whether the amphiphiles would help solubilise and better deliver this highly toxic compound. Our findings in this regard are highly preliminary and do support the theory that PAA amphiphiles of lower molecular weight can encapsulate and release DOX whilst mediating their entry into cells. In order to fully elucidate this potential more studies are required including stability, cellular uptake and more in depth cellular trafficking and response studies. For cancer nanomedicine we deemed that the longer molecular weight amphiphiles were not of use, however, this certainly does not discount their potential in other applications of drug delivery where prolonged release is desired over longer time periods. 


\section{Funding}

This work was funded by the Iraqi Ministry of Higher Education and Scientific Research (MOHSER).

\section{Competing Interests}

The authors would like to state that we have no competing interests.

\section{Supplementary Information}

Please see supplementary information for SI-Figures $1 \& 2$.

\section{Author Contribution}

JA \& AA carried out the laboratory work under the supervision of $\mathrm{CH}$ and $\mathrm{AC}$. $\mathrm{CH}$ wrote the manuscript and all authors approved of the final version before submission.

\section{References}

1. Hoskins, C, Ouaissi M, Lima S, Cheng W, Loureirio I, Mas E, Lombardo D, Cordeiro-Da-Silva A, Ouaissi A, Kong Thoo Lin, P. In vitro and In vivo Anticancer Activity of a Novel Nano-sized Formulation Based on Self-assembling Polymers Against Pancreatic Cancer. Pharm. Res 2010; 27(12): 2694-2703.

2. Nicholson JW. The Chemistry Of Polymers. 4th Edn. Cambridge: Royal Society of Chemistry;2012.

3. Barnett CM, Lees MR, Curtis ADM, Lin PKT, Cheng WP, Hoskins C. Poly(allylamine) Magnetomicelles for Image Guided Drug Delivery. Pharm Nanotechnol 2013;1:1-15.

4. Neha MD, Pranav BP, Anita PA, Vilasrau JK. Polymeric micelles as a drug carrier for tumor targeting. Chronicles of Young Scientists 2013;4(2):94-101.

5. Zhang, Y-R, Lin, R, Li, H-J, He, W, Du, J-Z, Wang, J. Strategies to improve tumor penetration of nanomedicines through nanoparticle design. WIREs Nanomed Nanobiotechnol. 2019; 11:e1519.

6. Hoskins C, Lin P, Tetley L, Cheng W. The Use Of Nano Polymeric SelfAssemblies Based On Novel Amphiphilic Polymers For Oral Hydrophobic Drug Delivery. Pharm Res 2012;29(3):782-794.

7. Hoskins C, Kong Thoo Lin P, Tetley L, Cheng WP. Novel fluorescent amphiphilic poly(allylamine) and their supramacromolecular self-assemblies in aqueous media. Polym Adv Technol 2012;23(3):710-719.

8. Thompson C, Ding C, Qu X, Yang Z, Uchegbu IF, Tetley L, Cheng WP. The Effect Of Polymer Architecture On The Nano Self-Assemblies Based On Novel Combshaped Amphiphilic Poly(Allylamine). Colloid Polym Sci 2008;286, 15111526.

9. Kim E-J, Kim S-H, Hyun Y-J, Noh Y-K, Jung H-O, Han S-Y, Park C-h, Choi BM, Noh G-J. Clinical and psychological characteristics of propofol abusers in Korea: a survey of propofol abuse in 38, non-healthcare professionals. Korean $\mathrm{J}$ Anesthesiol 2015;68(6):586-593.

10. Lin C, Symchowicz S. Absorption, Distribution, Metabolism, And Excretion Of Griseofulvin In Man And Animals. Drug Meta Rev 1975;4(1)75-95.

11. Zhao N, Woodle MC, Mixson AJ. Advances in delivery systems for doxorubicin. J Nanomed Nanotechnol. 2018;9(5):519. doi:10.4172/2157-7439.1000519. 
12. http://www.io.nihr.ac.uk/wp-content/uploads/migrated_new/10394-DoxorubicinNanoparticles.pdf [accessed 16/06/30].

13. Zhao M, Ding XF, Shen JY, Zhang XP, Ding XW, Xu B. Use of liposomal doxorubicin for adjuvant chemotherapy of breast cancer in clinical practice. J Zhejiang Univ Sci B. 2017;18(1):15-26. doi:10.1631/jzus.B1600303

14. Zhou, Q., Zhou, Y., Liu, X. Shen, Y. GDC-0449 improves the antitumor activity of nano-doxorubicin in pancreatic cancer in a fibroblast-enriched microenvironment. Sci Rep 2017; 7:13379.

15. Lu Y, Zhang E, Yang J, Cao Z. Strategies to improve micelle stability for drug delivery. Nano Res 2018;11(10):4985-4998.

16. Rehman N, Khan A, Bibi I,. Bica CID, Siddiq M. Intermolecular Interactions of Polymer/Surfactants Mixture in Aqueous Solution Investigated by Various Techniques, J Dispersion Sci Technol 2013;34(9):1202-1210.

17. Wong RSH, Dodou K. Effect of Drug Loading Method and Drug Physicochemical Properties on the Material and Drug Release Properties of Poly (Ethylene Oxide) Hydrogels for Transdermal Delivery. Polym 2017;9(12):286.

18. Varshosaz J, Hassanzadeh F, Sadeghi H, Firozian F, Mirian M. Effect of Molecular Weight and Molar Ratio of Dextran on Self-Assembly of Dextran Stearate Polymeric Micelles as Nanocarriers for Etoposide. J Nanomat 2012;265657.

19. Hussein YHA, Youssry M. Polymeric Micelles of Biodegradable Diblock Copolymers: Enhanced Encapsulation of Hydrophobic Drugs. Materials (Basel) 2018;11(5):688.

20. Manoharan Y, Ji Q, Yamazaki T, Chinnathambi S, Chen S, Ganesan S, Hill JP, Ariga $\mathrm{K}$, Hanagata N. Effect of molecular weight of polyethyleneimine on loading of $\mathrm{CpG}$ oligodeoxynucleotides onto flake-shell silica nanoparticles for enhanced TLR9mediated induction of interferon- $\alpha$. Int J Nanomedicine 2012;7:3625-35.

21. $\mathrm{Lu} \mathrm{X,} \mathrm{Wu} \mathrm{D}, \mathrm{Li} \mathrm{Z}$, Chen G. Polymer Nanoparticles. Prog Mole Biol Tran Sci 2011;104:299-323.

22. Phillips DJ, Pygall SR, Cooper VB, Mann JC. Overcoming sink limitations in dissolution testing:a review of traditional methods and the potential utility of biphasic systems. J Pharm Pharmacol 2012;64(11):1549_ 1559.

23. Hoskins C, Papachristou A, Ho TMH, Hine J, Curtis ADM. Investigation into Drug Solubilisation Potential of Sulfonated Calix[4]resorcinarenes. J Nanomed Nanotechnol 2016;7:2.

24. Ianiro A, García AG, Wijker S, Patterson SP, Esteves ACC, Tuinier R. Controlling the Spatial Distribution of Solubilized Compounds within Copolymer Micelles. Langmuir 2019;35(13)4776-4786.

\section{Figure Legends}

Figure 1. Chemical synthesis of PAA-Ox5.

Figure 2. Chemical characterisation of 1) PAA and 2) PAA-Ox5 (mw $15 \mathrm{kDa}$ ) using A) ${ }^{1} \mathrm{H}$ NMR spectroscopy carried out on $400 \mathrm{MHz}$ NMR at $25^{\circ} \mathrm{C}$ and $\mathrm{B}$ ) fourier transform infrared spectroscopy using FTIR-ATR, 64 scans.

Figure 3. Surface Tension results for PAA-Ox $515 \mathrm{kDa}$, $17.5 \mathrm{kDa}, \boldsymbol{\Delta} 120 \mathrm{kDa}$ and $\mathrm{X}$ $900 \mathrm{kDa}$ at $25^{\circ} \mathrm{C},(\mathrm{n}=3$, ave $\pm \mathrm{SD})$. 
Figure 4. Drug loading of propofol into PAA-Ox 5 at molecular weights of A) $15 \mathrm{kDa}, \mathrm{B}) 17.5$ $\mathrm{kDa}$ and C) $120 \mathrm{kDa}$ at $\mathbf{\square}: 1, \quad 5: 1$ and $\square 10: 1$ initial drug:polymer feed ratio and measured using high performance liquid chromatography $(\mathrm{n}=3, \pm \mathrm{SD}) . *$ denotes significant increase in propofol solubility compared with its intrinsic aqueous solubility $(\mathrm{p}<0.05)$.

Figure 5. Drug loading of griseofulvin into PAA-Ox 5 at molecular weights of A) $15 \mathrm{kDa}, \mathrm{B}$ ) $17.5 \mathrm{kDa}$ and C) $120 \mathrm{kDa}$ at $\mathbf{\square} 1$, 5:1 and 10:1 initial drug:polymer feed ratio and measured using high performance liquid chromatography $(\mathrm{n}=3, \pm \mathrm{SD}) .{ }^{*}$ denotes significant increase in grieofulvin solubility compared with its intrinsic aqueous solubility $(\mathrm{p}<0.05)$.

Figure 6. Drug release profiles of A) propofol from PAA-Ox 5 aggregates composed of 15 $\mathrm{kDa}, 17.5 \mathrm{kDa}$ and $120 \mathrm{kDa}$ PAA and $\mathrm{B}$ ) grisofulvin from PAA-Ox 5 containing $15 \mathrm{kDa}$ PAA. * denotes significant decrease in drug release compared to PAA-Ox5 at the same time point $(\mathrm{p}<0.005)$.

Figure 7. Evaluation of DOX formulations A) release profile over $24 \mathrm{~h}$ as measured using HPLC and B) cytotoxicity when exposed to pancreatic cancer cells for $24 \mathrm{~h}$, measured using the MTT assay $(\mathrm{n}=3, \pm \mathrm{SD}) .{ }^{*}$ denotes significant reduction in cell viability compared to free drug. 